\title{
Identificando áreas de actividad a través del uso de GPR en la costa del Soconusco
}

\section{Identification of Activity Areas Using GPR in Coastal Soconusco}

\author{
Marx Navarro Castillo \\ Universidad de Ciencias y Artes de Chiapas, México \\ HeCtor NeFF \\ Universidad del Estado de California, Long Beach, Estados Unidos
}

\begin{abstract}
Resumen: Este artículo se enfoca en la importancia de las técnicas para detección remota en los estudios arqueológicos, en específico el LIDAR, y de las técnicas geofísicas, como el georradar (GPR por sus siglas en inglés). El uso de LIDAR se ha popularizado en los últimos diez años y su empleo en lugares boscosos ha resultado muy eficaz a pesar de la poca visibilidad. En el caso del GPR generalmente se emplea en aquellos sitios donde se puede hallar evidencia de restos de estructuras. Aunque en los sitios identificados por el Proyecto Arqueológico Costa del Soconusco no se cuenta con edificaciones de dichas características, su utilidad para la identificación de áreas de producción cerámica ha sido determinante para el desarrollo de nuestro estudio. Esto nos ha permitido conocer más sobre los asentamientos ubicados en la región oriental del Soconusco.
\end{abstract}

Palabras Clave: Soconusco, georradar, prospección geofísica, detección remota, Lidar.

ABSTRACT: This article is focused on the importance of remote sensing techniques for archaeological studies, specifically LIDAR, and geophysics techniques, such as ground penetrating radar (GPR). The use of LIDAR has become popular in the last ten years and its use in forested places has been very effective despite the low visibility. In the case of GPR, it is generally used in those places where evidence of structure remains can be found. However, in the sites identified by the Project Costa del Soconusco there are no buildings with these characteristics, but their usefulness for the identification of areas of ceramic production have been decisive for the development of our study. These techniques have provided important data that has allowed us to know more about the settlements located in the eastern region of Soconusco.

KEYWORDs: Soconusco, ground penetrating radar, geophysical prospecting, remote sensing, LIDAR. 
Recibido: 30 de abril de 2019.

ACEPTACIÓn: 12 de agosto de 2019.

DOI: https://doi.org/10.19130/iifl.ecm.55.2020.0002

A finales de 2011 el Instituto Nacional de Antropología e Historia (INAH) otorgó el primer permiso de investigación al Proyecto Arqueológico Costa del Soconusco ${ }^{1}$ y desde esa fecha se han realizado estudios sistemáticos en la Costa Oriental del Soconusco (Figura 1), en específico en las zonas de manglares y esteros de la región. Nuestro proyecto se ha caracterizado por el empleo de diversas técnicas arqueológicas, además de las convencionales, como lo son el recorrido de superficie y las excavaciones arqueológicas, y ha puesto especial énfasis en la utilización de técnicas geofísicas de detección remota como lo son el magnetómetro y el radar de penetración terrestre (georradar o GPR, según sus siglas en inglés), así como de técnicas de detección remota, tal es el caso del LIDAR (Light Detection and Ranging).

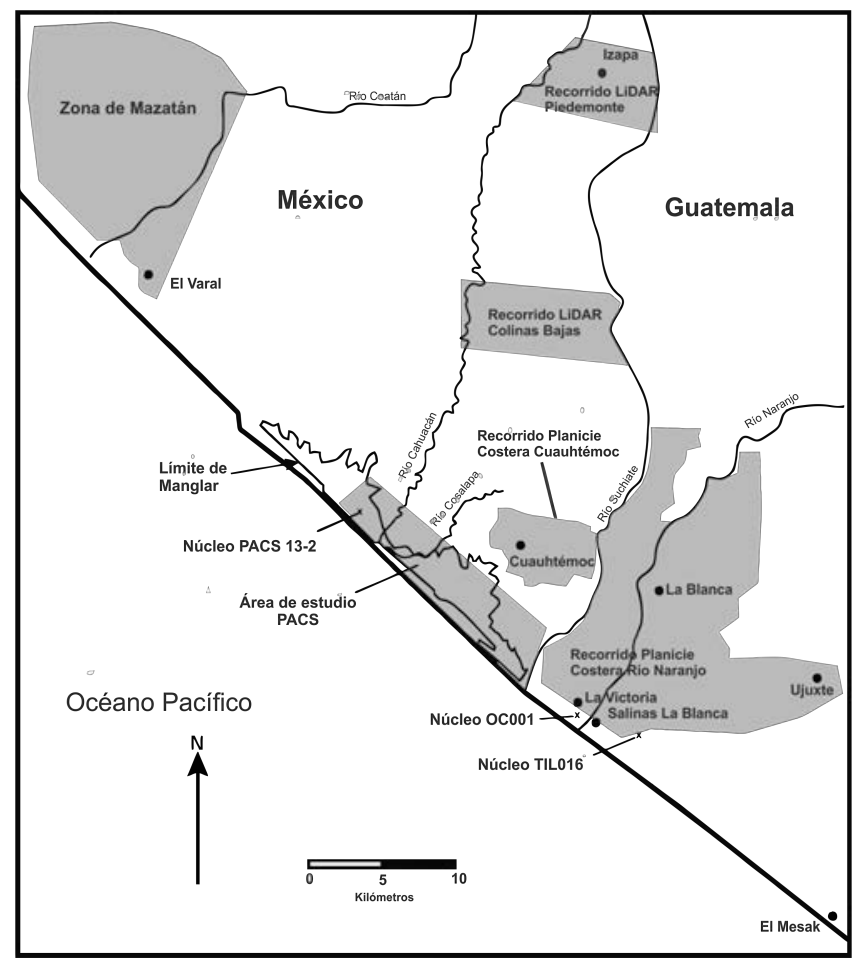

Figura 1. Mapa de la región este del Soconusco mostrando el área de estudio del PACs, así como otras áreas de reconocimiento arqueológico en Chiapas y Guatemala (Elaborado por Hector Neff).

\footnotetext{
1 En adelante PACs.
} 
El uso en conjunto de tales técnicas nos ha permitido realizar un trabajo de investigación más extenso, así como resultados más eficientes durante el desempeño de nuestras estancias de campo. Con base en este trabajo integral de investigación se ha logrado identificar un total de 216 montículos que se agrupan en ocho conjuntos dentro de un área de $54 \mathrm{~km}^{2}$ teniendo como límite sureste el río Suchiate, en la frontera internacional entre México y Guatemala. De los 216 montículos, se realizaron recolecciones superficiales y/o pruebas de pala en 113 , y se condujeron excavaciones sistemáticas en 15 de ellos. Las pruebas de pala consisten en una técnica donde se excava un área circular un poco mayor a la extensión de la cuchara de la pala, y cuyo objetivo es sondear el subsuelo para determinar si cuenta con elementos culturales y posteriormente comenzar con unidades de excavación.

\section{Antecedentes de investigación en la costa del Soconusco}

A partir de las primeras muestras tomadas durante el 2012, se estableció la secuencia cronológica de la ocupación. Así se logró identificar una ocupación constante desde el período Arcaico hasta períodos históricos. Como en otras regiones de la costa del Pacífico (Kennett et al., 2010; Neff et al., 2006), la evidencia más temprana consiste en modificaciones del paisaje durante el periodo Arcaico Tardío registradas en núcleos de sedimentos. Además de plantas que indican perturbaciones, se observó un grano de polen de maíz en niveles fechados alrededor de 2700 a.C. (Neff et al., 2018: 397). Los contextos arqueológicos más tempranos se asocian con fechas de aproximadamente 1600 a.C. (Neff et al., 2018: 399).

De manera más específica las investigaciones realizadas por el PACs han servido para confirmar que el período de mayor ocupación en la región de manglares, al sur del río Cahuacán, se llevó a cabo hacia el Formativo Tardío y Terminal. Durante este período, la zona producía grandes cantidades de sal para la población agrícola del interior. El Formativo Terminal fue seguido por una dramática caída poblacional alrededor del 100 d.C. y 200 d.C. Otro pico demográfico se observó durante el Clásico Terminal, cuando se desarrolló una especialización artesanal en la producción de la cerámica plomiza, tal vez el tipo cerámico más difundido durante dicho periodo, y uno de los tipos cerámicos de mayor difusión en la historia de Mesoamérica, siendo un fenómeno no sólo local o regional, sino pan-mesoamericano ya que se ha encontrado desde el occidente de México hasta Costa Rica, entre otros sitios intermedios (Shepard, 1948; Snarskis y Salgado, 1986; Mountjoy, 1991; Healy y Pohl, 1980; Fowler y Earnest, 1985; Kowalski et al., 1993; Jadot et al., 2019).

De la misma manera, nuestras investigaciones permitieron confirmar la propuesta inicial de Shook (1965), de que las áreas de producción del plomizo se encontrarían entre el río Naranjo de Guatemala y el río Coatán de Chiapas (Figura 1). Posteriormente, con base en estudios químicos de las fuentes de arcillas de 
dichos afluentes, Neff $(1984,1989,2002,2003)$ reafirmó la propuesta de Shook (1965), pero fue durante el desarrollo del proyecto actual que se identificaron de manera plena las áreas de producción de este tipo cerámico. Y se desecharon así propuestas anteriores sobre el origen de esta producción, como la Costa del Golfo y El Salvador, los cuales fueron considerados como lugares primarios (Medellín, 1960; Bruhns, 1980).

Como parte de los estudios emprendidos desde la primera temporada realizada por el PACS, se continuó con la investigación en la región y durante la tercera temporada de campo continuaron los estudios en los conjuntos Piñuela, Conquista Campesina y Cahuacán, que se encuentran al norte del río Cahuacán. En esta contribución nos enfocamos en los resultados obtenidos gracias al empleo de las técnicas de detección geofísica, específicamente el georradar, utilizado en el sitio identificado como Piñuela- $7,{ }^{2}$ con el objetivo de brindar información sobre su uso eficaz en la detección de anomalías y su posterior excavación en ambientes como los manglares. Los datos recabados reafirman la importancia de esta técnica de detección pues permiten hacer una primera prospección y a partir de ahí enfocarse en la confirmación in situ de su presencia y su carácter arqueológico, el cual nos compete.

\section{Estudios actuales}

El primer objetivo de nuestras investigaciones actuales ha sido aumentar y verificar el inventario de sitios arqueológicos dentro del área de estudio. Para completar dicha meta, se requirió de un método sistemático para localizar contextos arqueológicos dentro del manglar, una tarea que había sido imposible hasta muy recientemente, debido a la densidad del bosque y las condiciones pantanosas. Esto ha cambiado dramáticamente con la disponibilidad del LIDAR aéreo, que puede penetrar debajo del bosque para indicar las ubicaciones de los montículos, detectando incluso los más pequeños. La cobertura LIDAR en la zona de estudio fue realizada durante abril de 2011 , antes del inicio de nuestra primera temporada de campo y estos datos brindaron información del inventario de sitios (Neff, 2012).

La primera temporada de investigación se enfocó en la zona pantanosa al sureste del río Cahuacán. Con base en comparaciones con las colecciones de Izapa y varias publicaciones disponibles (Coe y Flannery, 1967; Lowe, Lee y Martínez, 1982; Love, 2002; Love, Popenoe y Escobedo, 2002; Voorhies, 1976), se concluye que las colecciones cerámicas de la primera temporada pertenecen principalmente a los períodos Formativo Tardío y Terminal [fases Frontera, Guillén y Hato en la cronología de Izapa (Lowe, Lee y Martínez, 1982)]. Se identificaron también seis montículos con indicaciones de ocupación de inicios del Formativo Temprano. Sorprendentemente, surgió la evidencia de una fuerte presencia del Clásico Tardío y

\footnotetext{
${ }^{2}$ En adelante PIN7.
} 
Posclásico Temprano en la planicie costera hacia tierra adentro, estando casi ausentes estos períodos en las áreas pantanosas ubicadas al sureste del río Cahuacán.

Las temporadas de 2013 hasta 2015 se enfocaron en el área al noroeste del río Cahuacán. Además de una alta cantidad de materiales procedentes del Formativo Tardío, muchos de los montículos de esta zona manifiestan en superficie densas concentraciones de cerámica plomiza, hallazgo que confirma la presencia de ocupación del Clásico Terminal y Posclásico Temprano dentro de la zona de pantanos.

Los estudios realizados nos proporcionaron también mucha evidencia sobre los usos de la zona pantanal del Soconusco durante el Formativo (Neff et al., 2018). Consideramos que dicha zona siempre ha sido un área industrial, donde los pobladores usaban la abundante madera del bosque de mangle como combustible para una variedad de actividades pirotecnológicas. Con base en el cálculo de las áreas de los distintos períodos, parece que la población creció gradualmente entre el Formativo Temprano y el Formativo Terminal. Mientras la población crecía, sus actividades parecen haberse desarrollado gradualmente hacia un rango más y más restringido, culminando en contextos especializados, probablemente la extracción de sal (RS3, RS23 y otros montículos del Formativo Terminal, como el Montículo Guzmán de Guatemala investigado por Nance, 1992). Las viviendas del Formativo Temprano muestran evidencia de que los antiguos habitantes realizaban varias actividades domésticas, pero alrededor del año 1000 a.C. la zona pantanal se transformó en una zona de especialización pirotecnológica, que creció en importancia hasta su apogeo durante el Formativo Tardío.

Las semejanzas entre la cerámica de este proyecto y la cerámica de Izapa sugieren que las poblaciones del pantano participaban en un sistema de interacción con los habitantes de Izapa a lo largo del período Formativo, y que las fluctuaciones demográficas de la zona pantanal se vinculaban con Izapa. La falta de evidencia de procesamiento de recursos alimenticios combinada con la evidencia ubicua de actividades pirotecnológicas sugiere el papel que la población del pantano desarrollaba para el abastecimiento de productos industriales para las poblaciones del interior. Estos productos podrían incluir la cerámica y, especialmente, la sal. Tal producto es particularmente importante porque cada ser humano, en este contexto, la necesitaba para sobrevivir, pues era el único modo de preservar carnes y pescados ante la ausencia de refrigeración. Desde este punto de vista, la zona pantanal evolucionó de manera complementaria con la de Izapa hacia un apogeo de población y especialización industrial durante el Formativo Tardío, época de máxima integración regional y mayor actividad constructiva y artística en Izapa.

\section{Los estudios de detección remota y geofísica}

Se pueden distinguir dos niveles de investigación arqueológica no-invasiva. Por un lado, hay un grupo de técnicas que se basa en sensores que detectan características de la superficie de la tierra desde una posición fuera de contacto con la 
misma, ya sea desde un avión o satélite. Probablemente la técnica más antigua de esta categoría usada en arqueología es la fotografía aérea. Esta técnica fue usada justo después de la primera guerra mundial y desde ese momento fue una herramienta importante para los arqueólogos. Sin embargo, es imperativo mencionar que la probabilidad de identificar sitios arqueológicos empleando fotografías aéreas verticales depende mucho de que la imagen haya sido tomada a una hora propicia, así como de la época del año, según la variación del sol en el cielo (Scollar et al., 1990: 26). Posteriormente, se desarrollaron diferentes técnicas de detección remota, como lo es la fotografía satelital, sobre todo la proporcionada por el Landsat Thematic Mapper. Dicha técnica es de mucha utilidad para identificar sitios arqueológicos en los humedales (Cox, 1992: 255). En Mesoamérica, Pope y Dahlin (1989: 89) la emplearon en el área maya, en específico en tres regiones: el norte de Belice, el sur de Quintana Roo y la cuenca superior del río Candelaria. Ello les permitió encontrar rasgos de antiguos canales en el área de estudio, los cuales pudieron haber estado vinculados con la agricultura. El LIDAR es la más reciente innovación dentro de tal categoría, y como se muestra en varias publicaciones recientes (Canuto et al., 2018; Rosenswig et al., 2013, Rosenswig, López-Torrijos y Antonelli, 2015; Chase, Chase y Weishampel, 2013), el impacto de esta nueva capacidad para detectar aun los más pequeños cambios de elevación está estimulando una verdadera revolución en la arqueología mesoamericana.

Otra categoría general de técnicas no-invasivas se basa en sensores que se mantienen en una posición de contacto o a pocos centímetros por encima de la superficie del suelo. Entre ellas se incluyen la conductividad, la resistividad, la magnetometría y el georradar. En Mesoamérica, la búsqueda de monumentos de basalto por magnetometría en el sitio olmeca de San Lorenzo (Breiner y Coe, 1972) fue uno de los primeros usos de esta clase de técnica. Más recientemente el georradar ha proporcionado resultados útiles en una variedad de contextos (Valdés y Kaplan, 2000; Safi et al., 2012), siendo una de sus ventajas que proporciona evidencia de las características del subsuelo en distintas profundidades.

\section{Métodos}

En lo que compete al presente trabajo, las técnicas empleadas fueron el LIDAR para la detección de sitios y el georradar para la detección de características del subsuelo. Los datos de LIDAR para este proyecto se generaron por Airborne 1 Corporation durante el mismo período en que el Proyecto de Reconocimiento Regional Izapa (PRRI) recolectó datos para su área de estudio (Rosenswig et al., 2013; Rosenswig, López-Torrijos y Antonelli, 2015). El área de cobertura se extendió $17 \mathrm{~km}$ al noroeste de la frontera con Guatemala, de la playa hasta un poco más de $3 \mathrm{~km}$ hacia el interior (Figura 1). La mayor parte de esta área de 54 
$\mathrm{km}^{2}$ consiste en densos bosques de mangle, lo cual impide hacer un recorrido de superficie sistemático. De tal manera que, con las imágenes de LIDAR, como ya se mencionó, fue posible identificar 216 montículos, incluso algunos de ellos con alturas menores a los $50 \mathrm{~cm}$.

El georradar (Figura 2), por su parte, emite ondas de frecuencia radio en contacto con el suelo y detecta la amplitud de las reflexiones durante un lapso fijo de tiempo. Para aplicaciones arqueológicas, como la nuestra, la antena más útil es de una frecuencia de $400 \mathrm{MHz}$. Esta antena registra la llegada de ondas reflejadas durante 60 nano-segundos (ns) que, con condiciones de suelo como en los montículos del área del PACS, corresponde a una profundidad de aproximadamente $1.8 \mathrm{~m}$. El radargrama manifiesta cambios de amplitud de las ondas reflejadas que indican cambios de propiedades eléctricas (conductividad y resistividad), las cuales pueden ocurrir en pisos, alineaciones de piedra, cavidades y otras clases de variaciones en los materiales ubicados debajo de la superficie. Se ha empleado este tipo de estrategia de manera exitosa en otros estudios (Chinchilla et al., 2008; Daniels et al., 2012; Safi et al., 2012).

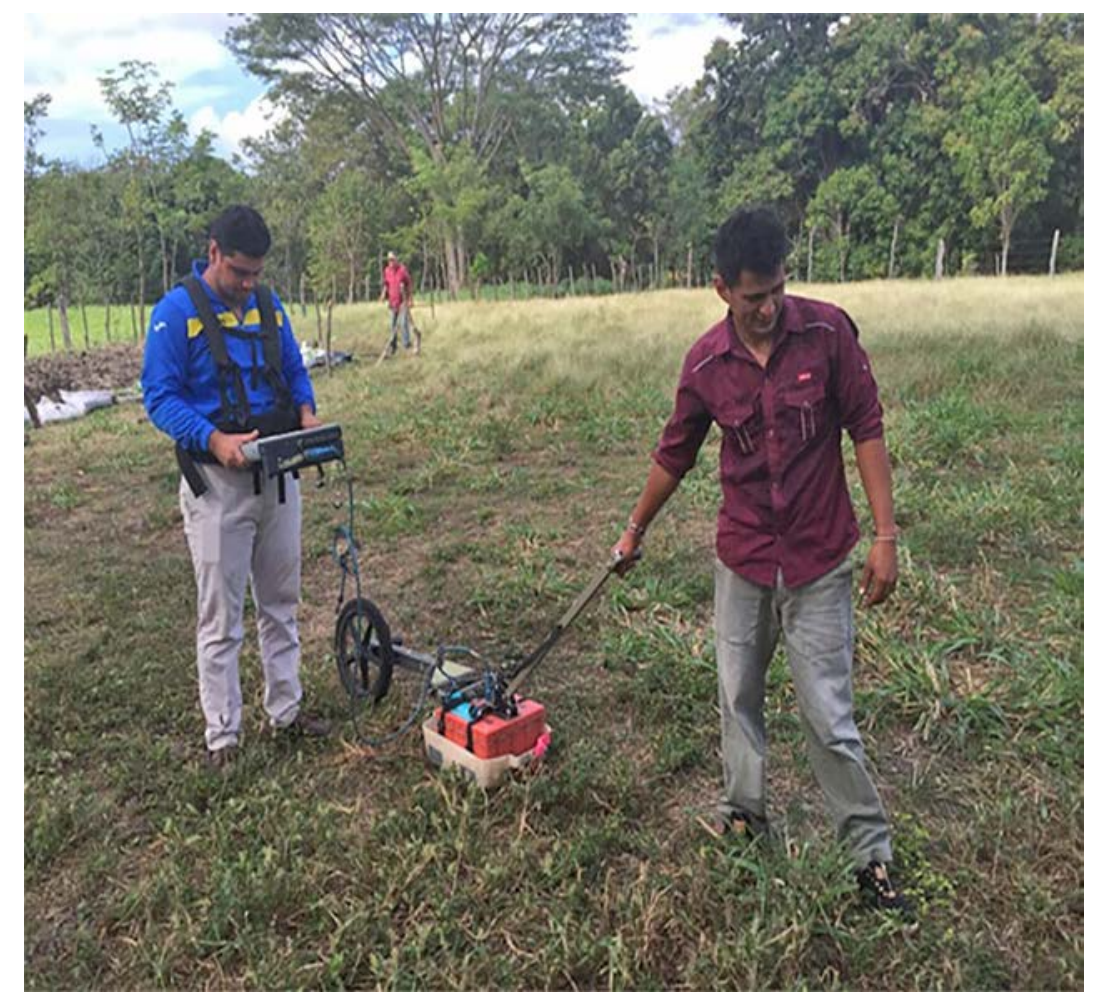

Figura 2. Prospección con el georradar. Una persona jala la antena mientras que la otra carga la computadora que está registrando los radargramas a lo largo de la línea de prospección (Fotografía de Hector Neff). 
Se procesaron los radargramas, que indican variación vertical debajo del aparato, por medio del programa GPR-Slice v. 7.0 (Goodman, 2017). Este programa une todos los perfiles de radar del área de prospección para hacer un modelo tridimensional de las reflexiones. En otras palabras, para cada cuadrícula de prospección, tenemos un volumen cúbico que se extiende entre 1.6 y 1.9 m debajo de la superficie. Aunque hay una cantidad de opciones diferentes para visualizar y presentar los datos, una de mucha utilidad para aplicaciones arqueológicas consiste en crear una serie de mapas que representan reflexiones en diferentes tiempos (el tiempo corresponde a la profundidad). En la prospección de los montículos del pacs se procesaron los datos de los 60 ns (una ventana de tiempo efectivo de $55.55 \mathrm{~ns}$ ) en 12 distintos cortes de $6.21 \mathrm{~ns}$, que representan aproximadamente $21 \mathrm{~cm}$. Es así que se procesaron un total de 12 mapas por cada cuadrícula prospectada.

Debido a que el GPR es capaz de identificar anomalías relacionadas con intenso calor (en este caso podría tratarse de la producción cerámica o la extracción de sal), dichas anomalías fueron útiles para determinar con mayor certeza los lugares donde se ubicarían las unidades de excavación. Es así que la prospección geofísica antes de la excavación aumenta la información proporcionada durante la investigación, porque hace más probable el identificar áreas de incendios, basureros u otras clases de restos que se asocian específicamente con actividades humanas del pasado.

Después de la recolección sistemática y el manejo de los datos proporcionados por el GPR se colocaron unidades de excavación de $2 \times 2 \mathrm{~m}$ sobre algunas de las anomalías detectadas. Muestras pequeñas de sedimentos para análisis micro-arqueológico fueron recolectadas de cada nivel, las cuales fueron obtenidas a través del muestreo de suelo y debido a su tamaño únicamente posibles de recolectar de esta manera. Además, se obtuvieron pedazos intactos de carbón para determinación de fechas a través de Carbono-14, que se tomaron in situ, registrando sus coordenadas tridimensionales.

\section{Resultados}

Para ilustrar la eficacia del LIDAR en combinación con el GPR, el resto del artículo se enfoca en el área al noroeste del río Cahuacán, donde realizamos investigaciones entre 2013 y 2015. La Figura 3 muestra la imagen LIDAR donde se puede ver la mayor parte de tres complejos de montículos, que denominamos el Complejo Piñuela, el Complejo Conquista Campesina y el Complejo Cahuacán. Durante mayo de 2013 realizamos nuestras primeras investigaciones en los montículos del Complejo Piñuela, incluyendo los siete que aparecen en la Figura 4. La labor inicial de este trabajo fue la limpieza de la vegetación para posteriormente proceder con la recolección de materiales en superficie, pruebas de pala y prospección con el GPR en algunos de los montículos, incluyendo PIN7, en el cual nos enfocamos aquí. 


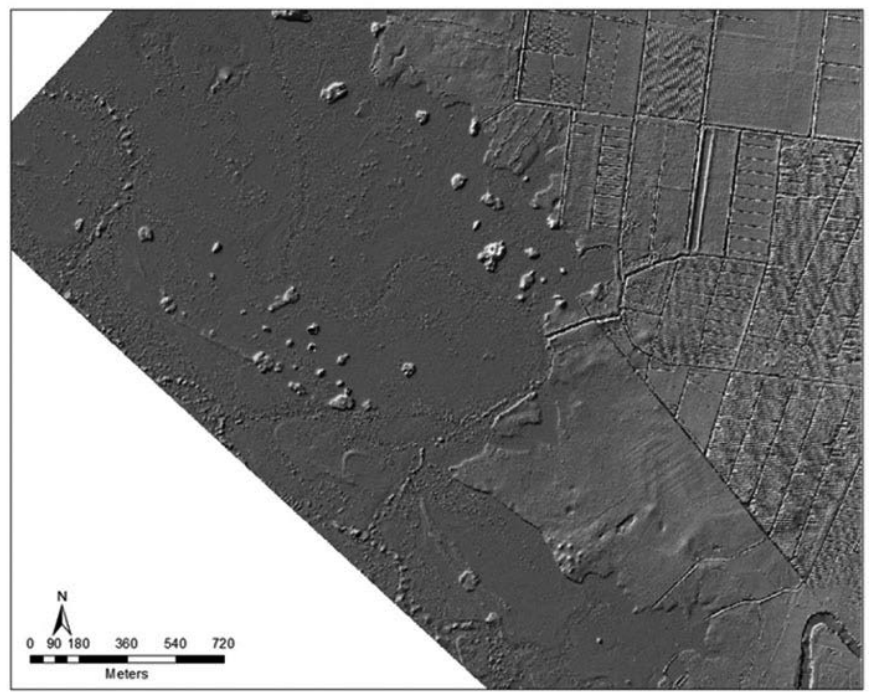

Figura 3. Plano de la esquina noroeste del área de estudio del pacs mostrando partes de los complejos de montículos Conquista Campesina, Piñuela y Cahuacán. Se puede ver parte del río Cahuacán al extremo sur de la imagen. La sección este muestra rasgos de una plantación de plátanos que se encuentra hacia el interior de la zona de mangle (Realizado por Hector Neff).

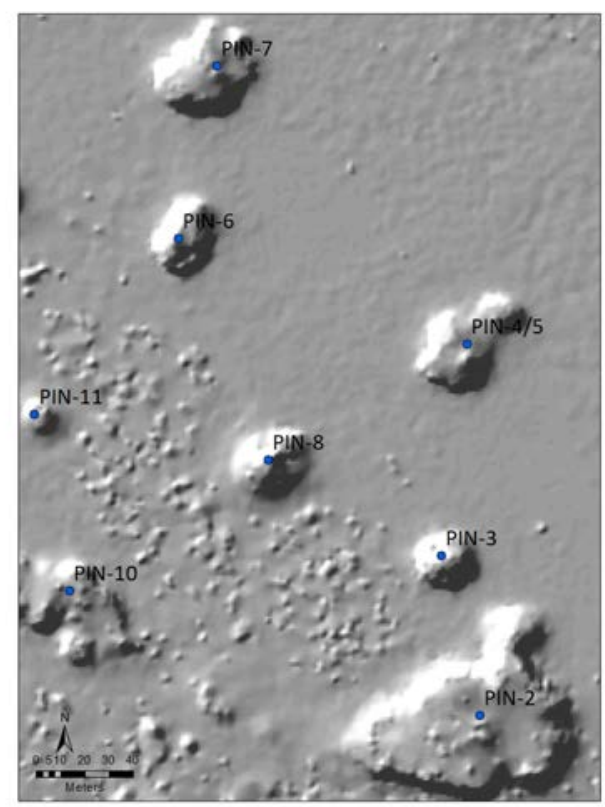

Figura 4. Imagen LIDAR del centro del complejo de montículos denominado Piñuela (Elaborado por Hector Neff). 
PIN7 es un montículo de forma ovoide, con su eje mayor, de aproximadamente $45 \mathrm{~m}$, orientado de noreste hacia suroeste. Tanto la recolección sistemática de superficie como las pruebas de pala realizadas durante mayo de 2013 indicaron ocupaciones del Formativo Tardío y el Clásico Terminal. Una prueba de pala en la orilla oeste del montículo encontró una densidad increíble de cerámica plomiza, y con base en ello decidimos planear investigaciones más extensivas, con el interés de recuperar mayor evidencia de la producción de este famoso tipo cerámico. Durante mayo de 2013 realizamos prospección con el GPR, y en julio de 2014 se comenzaron las excavaciones sistemáticas.

La prospección del GPR en PIN7 cubrió dos cuadrículas, con un área total de $317 \mathrm{~m}^{2}$. Se ubicaron las dos unidades en el centro de la parte alta del montículo con una orientación paralela al eje largo del montículo, usando un sistema de cuadrículas arbitrarias para registrar la posición del aparato. La orientación de la prospección fue de oeste a este, es decir, a lo largo del eje mayor de cada unidad. Como se mencionó previamente, se procesaron los datos con el programa GPR Slice v. 7.0 (Goodman, 2017). Con la ventana total de tiempo de $60 \mathrm{~ns}$, hubo una ventana efectiva de $55.55 \mathrm{~ns}$, que se dividió en 12 cortes de $6.21 \mathrm{~ns}$ (con un traslape de $20 \%$ ), cuyo cálculo equivaldría a $21.7 \mathrm{~cm}$ de profundidad, tomando en cuenta que la velocidad del radar en el suelo es de $7 \mathrm{~cm} / \mathrm{ns}$. Con estos ajustes y suposiciones se calcula que el GPR alcanzó una profundidad de $194 \mathrm{~cm}$.

La Figura 5 es el radargrama que empieza en la esquina noroeste del área de prospección, en la posición $\mathrm{x}=989, \mathrm{y}=997$. Se puede notar que hay una serie de reflexiones intensas entre $\mathrm{x}=993$ y $\mathrm{x}=995$ que continúan a través de varios centímetros de profundidad. Cuando se unen los 38 radargramas de la prospección total, los doce cortes forman una serie de mapas de reflexiones producidas en diferentes profundidades. La Figura 6 muestra los 12 mapas que resultaron de dicha operación. Estos mapas indican que la anomalía más consistente y más profunda de toda el área de la prospección se centra en $\mathrm{x}=994, \mathrm{y}=997$, correspondiendo a las reflexiones intensas visibles en la Figura 5.

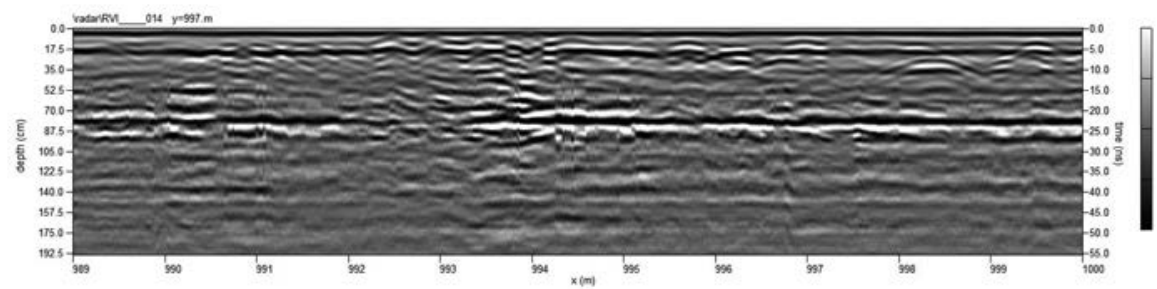

Figura 5. Radargrama de la línea y = 997, en la esquina noroeste del área de prospección (Realizado por Hector Neff).

En nuestra experiencia con otras aplicaciones de GPR en Mesoamérica (por ejemplo, Safi et al., 2012), el GPR ha sido eficaz en la detección de alineamientos 


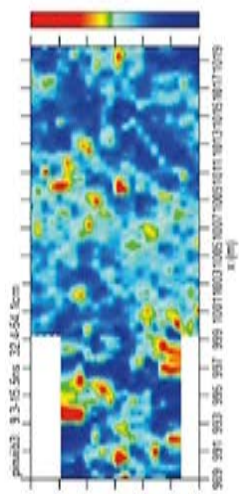

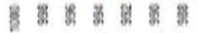

(iii) 1

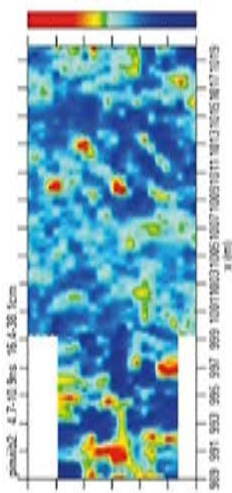

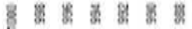

(iii) $A$

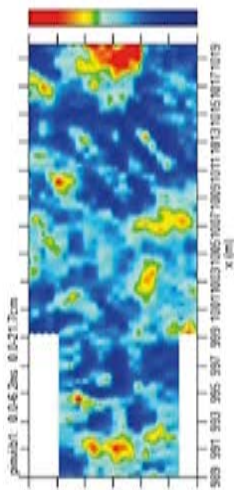

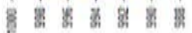

(wi) $k$

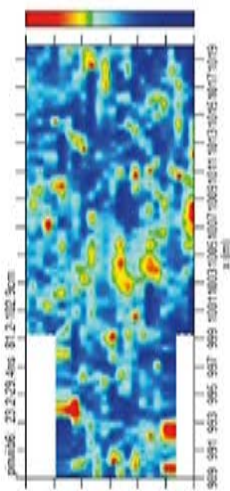

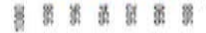

(iii) $\lambda$

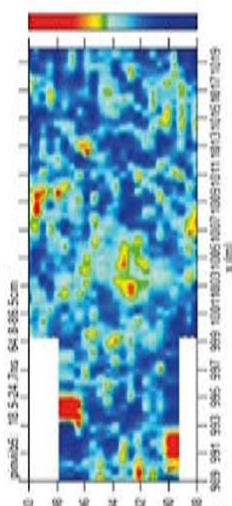

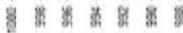

(ii) A

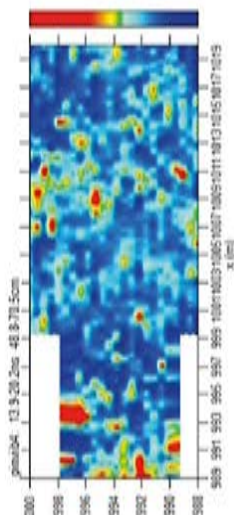

(iw) $\bar{k}$

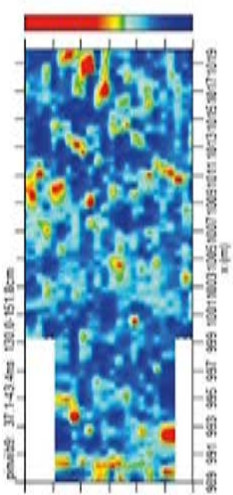

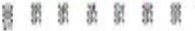

(ini) $A$

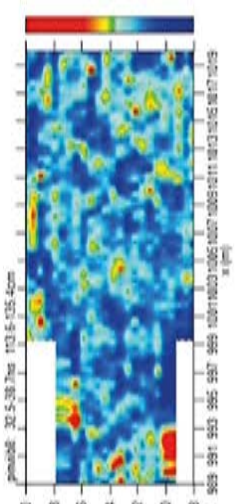

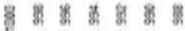

(wiv) $A$

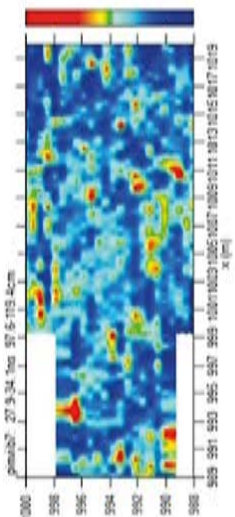

(iw) $\hat{k}$

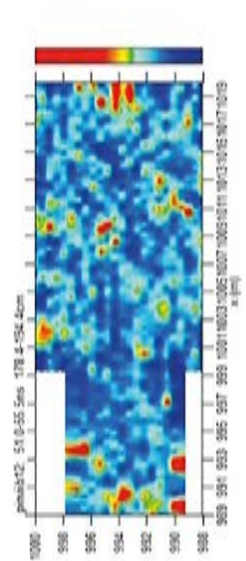

(iii) $A$

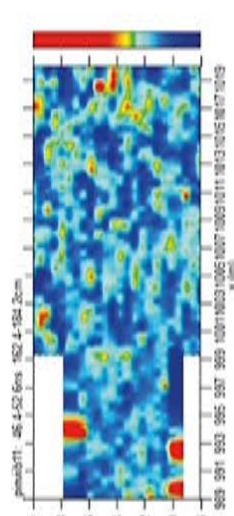

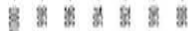

(iii) $\mathrm{A}$

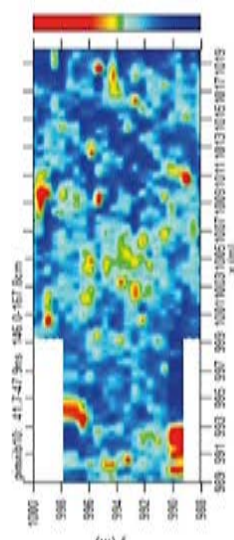

(iii) $\mathrm{A}$

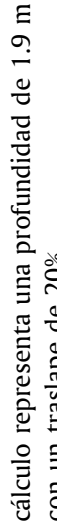

引

尫

은

돈

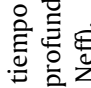

ษั

总 छัญ

产京

䒿 苋

ช

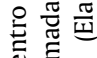

के

弐

离 芭

¿

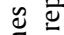

㐫

氖

을

壳茊

ㄹ

$\stackrel{0}{0}$

ช

ษั

6

茪 
de piedra y ubicaciones de estructuras. En el caso de PIN7 y otros sitios en el estudio de PaCs, casi no se encuentran piedras, tampoco son obvios los patrones rectangulares que podrían indicar delimitaciones de estructuras (Figura 6). No obstante, los resultados de la prospección del GPR en PIN7 junto con los resultados de la recolección de superficie, las pruebas de pala y la configuración topográfica del montículo nos motivaron a implementar excavaciones sistemáticas para la temporada de julio de 2014. La Figura 7 indica la ubicación de las tres excavaciones sobrepuestas en el mapa del corte 4 del GPR.

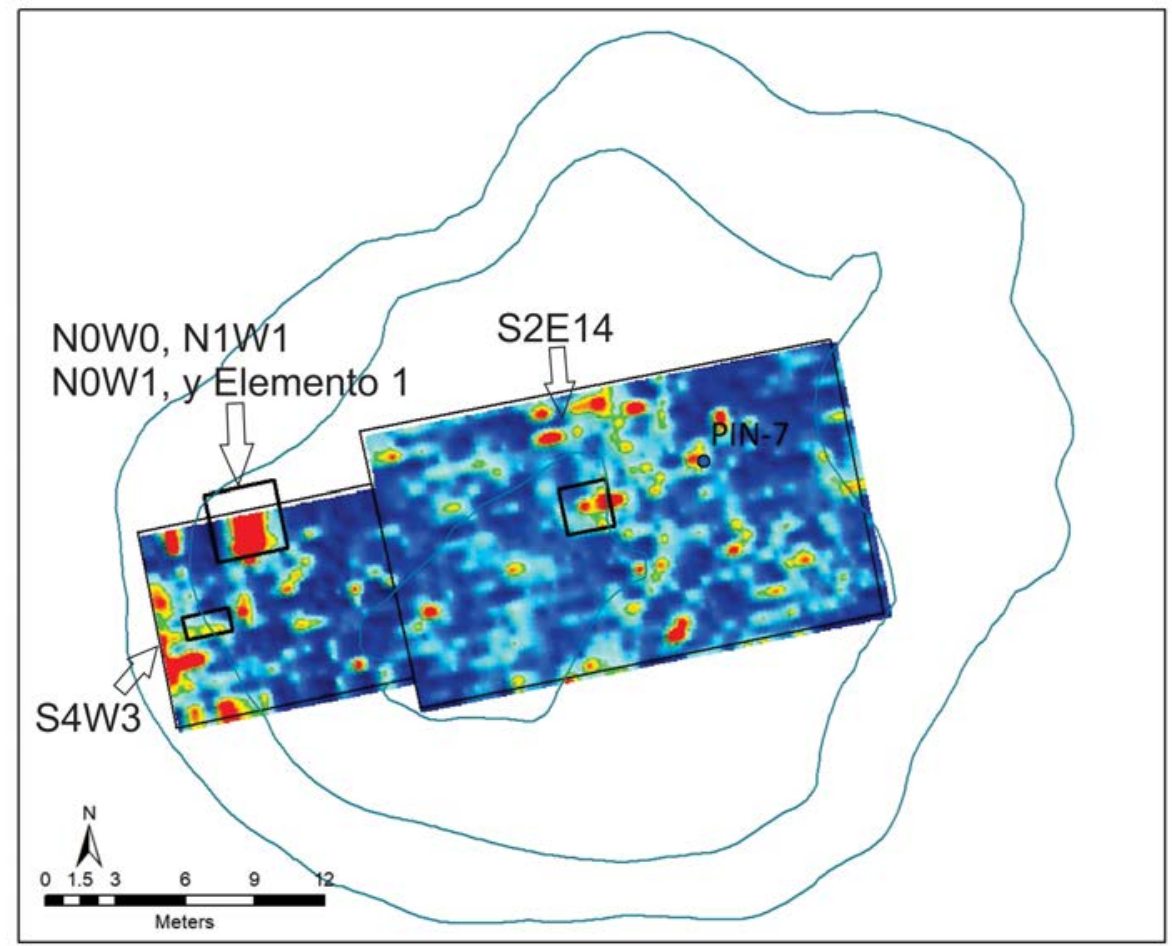

Figura 7. Corte 4 de la prospección del georradar en PIN7 sobrepuesto al mapa topográfico del sitio obtenido del LIDAR. La imagen muestra también la ubicación de las tres excavaciones que se discutirán más adelante (Obtenido por Hector Neff).

\section{Excavaciones}

Como se mencionó en la sección de métodos, las unidades de excavación fueron ubicadas con base en las anomalías observadas por el georradar. Posteriormente se hizo un sistema de cuadrículas orientado de manera paralela a las cuadrículas de prospección del GPR, con el fin de llevar un mayor control sobre las unidades excavadas además de que nos permitía ubicar y asociar mejor los contextos exca- 
vados con los sondeados a través de GPR. Para los objetivos de este artículo no es necesario analizar las excavaciones estrato por estrato sino únicamente aquellos niveles donde el GPR identificó anomalías.

NOW0, N1W1, N2W0, N2W1 y Elemento 1

Una de las excavaciones, NOW0, se empezó en la ubicación de las fuertes reflexiones del GPR en las coordenadas $\mathrm{x}=994, \mathrm{y}=997$ del sistema de cuadrículas de la prospección y esto nos indicó la posibilidad de que la anomalía GPR fuera reflejo de actividades de producción cerámica. Como se mencionó previamente, la unidad NoW0 fue colocada en el lugar donde se observaron unas reflexiones fuertes del GPR que empezaron a una profundidad de $60-80 \mathrm{~cm}$ (Figuras 6 y 7). Comenzó como una unidad de $2 \times 2 \mathrm{~m}$, pero las extensiones eventualmente alcanzaron un tamaño de $3 \times 3 \mathrm{~m}$. Las unidades fueron excavadas empleando niveles métricos arbitrarios de $20 \mathrm{~cm}$, con la finalidad de llevar un control tanto de las capas estudiadas como del material recolectado.

En esta unidad de excavación el suelo era poco compacto con una textura limosa y con algunas intrusiones de piedras de color café. En general se encontró evidencia de cilindros sólidos de cerámica, así como también de tiestos en la esquina suroeste de la unidad. Es importante señalar que los cilindros habían sido identificados previamente y siempre se encontraban asociados con densidades elevadas de cerámica plomiza, lo que nos hace pensar que eran herramientas usadas en la fabricación de dicha cerámica, ya que pudieron haber servido como soportes de las vasijas o bien como parte de las paredes de hornos incipientes. Esta propuesta está basada en la evidencia observada en otras áreas estudiadas por el PaCs, como lo es Conquista Campesina, en específico en el sitio Conq18 donde se encontraron varios cilindros en posición vertical dentro de un rasgo utilizado para la cocción cerámica, apoyando así la noción de que formaban parte de las paredes de hornos. Asimismo, los cilindros con pequeñas muescas pudieron haber sido utilizados como separadores entre las vasijas.

A una profundidad de $60 \mathrm{~cm}$ aproximadamente se identificó un suelo amarillento bastante compacto, y de igual manera la densidad cerámica aumentó significativamente, sobre todo en cuanto a la cerámica plomiza. Sin embargo, no fue hasta los $80 \mathrm{~cm}$ que se observó un rasgo circular que concuerda en forma y profundidad con lo mostrado por el GPR. Este elemento estaba constituido por un depósito de cal solidificada, probablemente de ceniza de madera, que prácticamente formaba un círculo perfecto. Debido a las características intrínsecas del elemento, al tratarse de ceniza, el GPR fue capaz de identificarlo ya que la dureza de las paredes y la densidad interior del depósito contrastaron con el suelo que lo rodeaba. A este rasgo se le denominó Elemento 1 de la Unidad PIN 7.

Además de la característica circular que se identificó al inicio del Elemento 1, también se observó un cambio en la matriz ya que era limo-arenosa y de un color negro, como si se hubiera llevado a cabo la quema de algo. Asimismo, hubo un 
incremento notable en cuanto a la densidad de cerámica y la presencia de cilindros sólidos, lo que apunta a que podría tratarse de alguna especie de horno. Sin embargo, no ha sido hallada evidencia de un suelo rojizo como en otras áreas estudiadas por este proyecto e identificado en la Figura 7.

Posteriormente, a una profundidad aproximada de $1.3 \mathrm{~m}$ se encontró un entierro de tipo primario, en el cual el individuo fue colocado de manera directa sobre el suelo. El entierro fue enviado a la Facultad de Ciencias Antropológicas de la Universidad Autónoma de Yucatán para su respectivo estudio y análisis, el cual fue conducido por el equipo de los doctores Tiesler y Cucina. El cuerpo estaba en una posición flexionada, y con base en la extensa investigación se puede señalar el colapso de los segmentos (poco movimiento de la cintura escapular y giro del cráneo), que probablemente indican que el individuo estuvo envuelto al momento de la sepultura y que fue colocado en un espacio rellenado. El personaje fue puesto en decúbito dorsal, con las extremidades superiores parcialmente flexionadas sobre la pelvis y las piernas completamente flexionadas. ${ }^{3}$ De igual manera, el trabajo osteológico de la Universidad Autónoma de Yucatán nos menciona que debió tratarse de una persona de sexo masculino y con base en la superficie auricular se calcula su edad entre los 25 y 29 años. ${ }^{4}$ A partir de los trabajos de datación realizados en el Laboratorio de Radiocarbono AMS de Institutes of Energy and the Environment de Pennsylvania State University, el individuo tiene una datación calibrada de 1175 a.p. $+-25 .{ }^{5}$ Ello apunta hacia una ocupación asociada con el Clásico Terminal en la costa oriental del Soconusco.

S4W3

La excavación S4W3 se ubicó dentro de un área que parecía una formación rectangular en los primeros cortes del GPR, pero esta localidad también se escogió por su proximidad con la prueba de pala que localizó grandes cantidades de cerámica plomiza. Las anomalías identificadas fueron similares a aquellas de NOW0. Esta unidad se encontraba a las faldas de PIN 7.

En el segundo nivel de la excavación, los sedimentos se convirtieron en una tierra muy obscura, con una alta densidad de cerámica plomiza y cilindros sólidos. Esta capa oscura también era bastante húmeda. El cambio en la constitución del subsuelo probablemente fue la característica detectada por el GPR que arrojó una anomalía. En la sección este de la excavación, la capa oscura tuvo una profundidad de aproximadamente $30 \mathrm{~cm}$, y por debajo se encontró una capa compactada, de color amarillo. En la parte superior de la capa amarilla, en la esquina noreste, se encontró un conjunto de cilindros sólidos (Figura 8), parecidos a otros que se han encontrado en posibles contextos de fabricación del plomizo, inclu-

\footnotetext{
${ }^{3}$ Vera Tiesler, comunicación personal, 2017.

${ }^{4}$ Vera Tiesler, comunicación personal, 2017.

5 Brendan Culleton, comunicación personal, 2017.
} 
yendo NOW0 de PIN7 (véase arriba). Dentro de la capa amarilla, por debajo del conjunto de cilindros, desaparecen tanto el plomizo como los cilindros sólidos, y la cerámica consiste de puros tipos del Formativo Tardío.

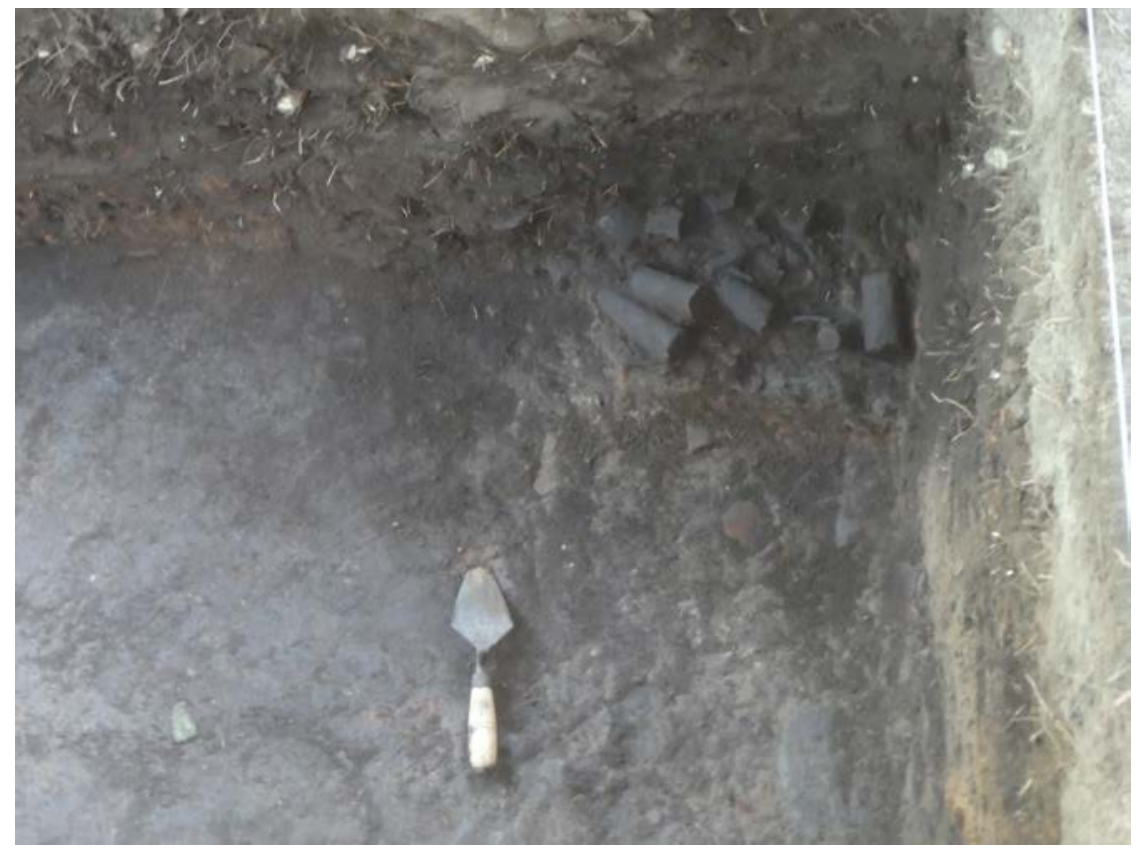

Figura 8. Foto de la esquina noreste de la excavación S4W3, nivel 3, mostrando el conjunto de cilindros sólidos que se encuentra a la base de la capa oscura (Fotografía de Marx Navarro Castillo).

La estratigrafía de S4W3 confirma que hay un depósito del Clásico Terminal con mucho plomizo y sedimentos carbonizados encima de los niveles del Formativo Tardío, sin ocupación de los períodos intermedios. Cuando los pobladores del Clásico Terminal rehabilitaron el sitio, sus actividades se realizaron en una superficie constituida de artefactos de la ocupación anterior, tales como material orgánico y suelos que se desarrollaron durante los $\sim 800$ a 1000 años de abandono. Una de las primeras actividades que realizaron los nuevos pobladores incluyó el uso de los cilindros sólidos en una actividad pirotecnológica e inferimos que fue en relación con la producción de la cerámica plomiza.

Resulta interesante mencionar que la investigación produjo evidencia directa del reuso de fragmentos de cerámica del Formativo Tardío en actividades pirotecnológicas del Clásico Terminal. En el nivel 3 de la excavación se encontró un fragmento grande de cerámica del Formativo Tardío cubierto completamente por una capa de concreto formado por la interacción de carbonatos de ceniza de madera con silicatos del suelo y agua (Figura 9). La presencia de fragmentos de carbón dentro del carbonato nos ofreció la oportunidad de fechar esta capa de carbona- 
tos a través del $\mathrm{C}^{14}$ en el ambiente del Soconusco, para lo cual entregamos ambos al Laboratorio de Radiocarbono AMS de Institutes of Energy and the Environment de Pennsylvania State University. Como se aprecia en la Figura 10, mientras que el fragmento de carbón proporcionó una fecha consistente con el Formativo Tardío, la capa de concreto es casi igual a la fecha del Clásico Terminal del esqueleto del Elemento 1, aunque con un error más pequeño. En otras palabras, los pobladores del Clásico Terminal usaron este fragmento cerámico dentro de una instalación pirotecnológica que produjo grandes cantidades de ceniza de madera, las cuales cubrieron este tiesto entre otros; por consecuencia de actividades posteriores al incendio, un pedazo de carbón restante de la primera ocupación (Formativo Tardío) fue incrustado en el concreto del Clásico Terminal.

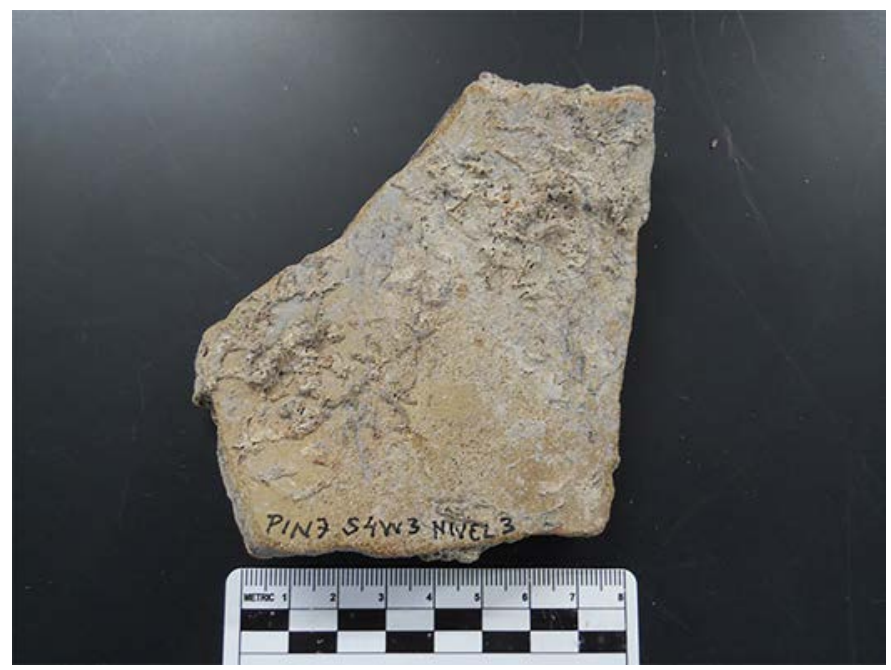

Figura 9. Tiesto cerámico con restos de calcita solidificada, del cual se obtuvieron las muestras de carbón y carbonato que proporcionaron las dos fechas de $\mathrm{C}^{14}$, Formativo Tardío y Clásico Terminal, discutidas en el texto (Fotografía de Brendan Culleton).

PIN7 Radiocarbon Dates

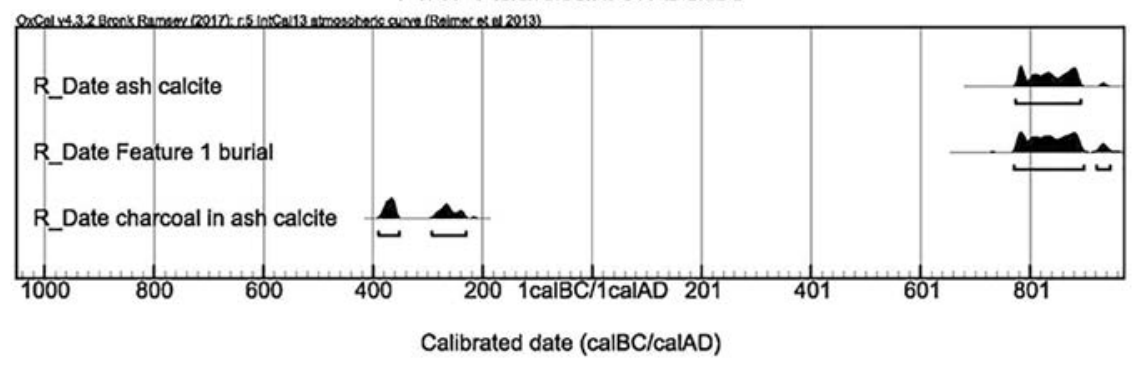

Figura 10. Datación por $\mathrm{C}^{14}$ de calcita en tiesto cerámico, así comol de carbón identificado en la unidad de excavación S4W3 (Gráfica realizada por Brendan Culleton). 
Se colocó la unidad S2E14 en un área abierta, donde se observaron en los mapas del GPR reflexiones bastante altas en varias profundidades (Figuras 6 y 7). Esta posición coincide con la parte más alta del montículo. En contraste con el primer conjunto de excavaciones, se encontraron muy pocos ejemplos de cerámica plomiza en los primeros niveles de excavación. Asimismo, hubo una ausencia de cilindros sólidos. La cerámica consistió casi puramente de tipos del Formativo Tardío, de pasta roja y paredes gruesas, parecidas al fragmento del cual obtuvimos las dos fechas de $\mathrm{C}^{14}$ discutidas previamente.

Como en las otras excavaciones, esta operación también produjo evidencias de actividades de cocción, pero no son parecidas a las encontradas en aquellas unidades, ya que en el caso de S2E14 el elemento estuvo compuesto por paredes de arcilla de un color rojizo/naranja. Dicho color nos apunta a actividades pirotecnológicas, probablemente la producción de sal obtenida a partir de la técnica de sal cocida y/o a la producción de vasijas cerámicas para contener la salmuera para hervir durante este proceso productivo. La deposición de tales estratos podría sugerir un reuso de esta área para el quemado de la arcilla, ubicándose los contextos uno encima del otro durante el transcurso del tiempo debido a la producción constante de sal y cerámica (Figura 11).

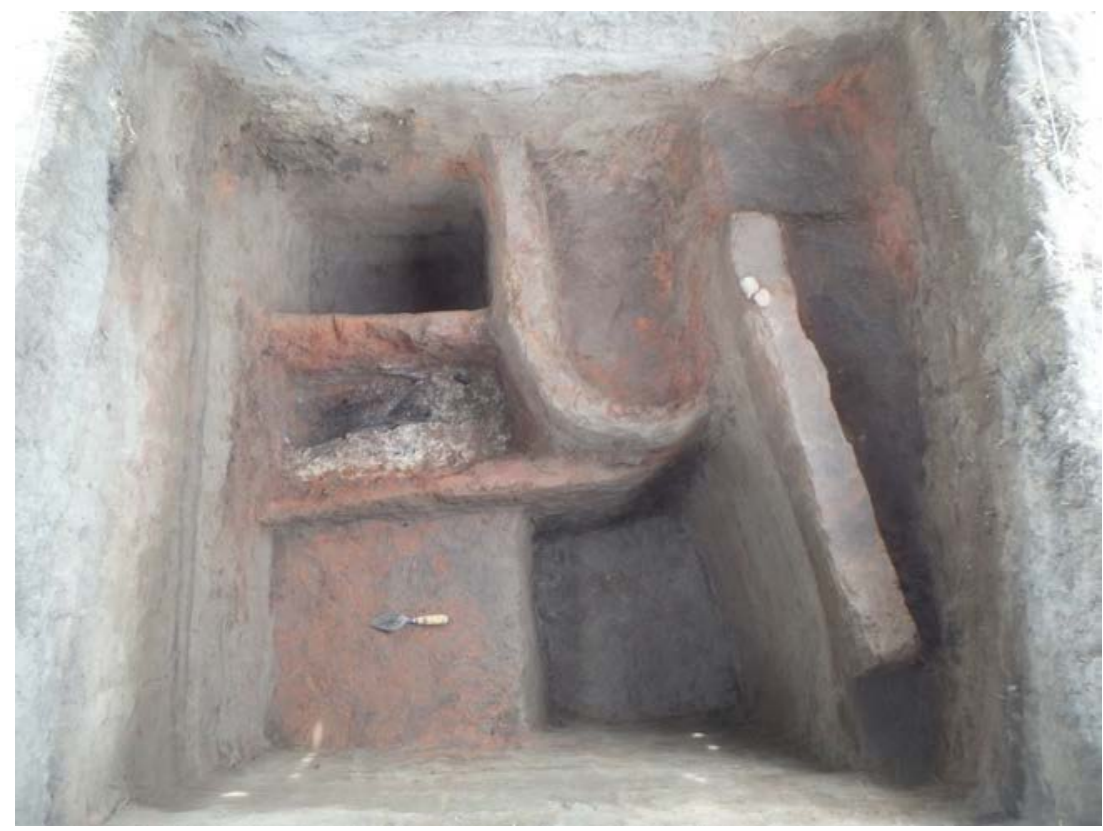

Figura 11. Unidad de excavación S2E14. Se logra observar el color rojizo del suelo, así como también los restos de ceniza solidificada característica de esta unidad

(Fotografía de Marx Navarro Castillo). 


\section{Conclusiones}

Durante las temporadas que el PACs ha realizado desde 2011 se han empleado técnicas tanto tradicionales como innovadoras, lo cual nos ha servido para tener una mejor recolección de datos, así como su registro. Las técnicas de detección remota como el LIDAR, al igual que las geofísicas, como el GPR, han sido de gran utilidad, según se ha demostrado a lo largo de este texto, para planificar el desarrollo del proyecto. El primer paso fue la recolección de datos a través de LIDAR, y con base en ello fue posible identificar 216 montículos en una región que nunca antes había sido estudiada de manera sistemática; gracias a este primer acercamiento pudimos elegir cuál de los sitios era posible estudiar in situ. Posteriormente, a través de las técnicas geofísicas, como el georradar, fue posible identificar anomalías en el subsuelo, lo cual resultó de gran ayuda para ubicar las áreas de excavación con mayor certeza, ya que no se realizaron de manera aleatoria sino con base en los datos arrojados por dicha herramienta. Asimismo, pudimos confirmar que el georradar no solamente es de utilidad en la detección de alineamientos de piedra o de ubicación de estructuras, sino también en la identificación de anomalías asociadas con las actividades pirotecnológicas, como suelos expuestos a altas temperaturas, característica principal de dicha labor artesanal.

El uso de las técnicas presentadas en este artículo ha resultado eficaz para nuestro estudio, ya que ha permitido identificar áreas propicias para su excavación con base en las anomalías observadas en el subsuelo. A partir de las excavaciones posteriores fue posible conocer más sobre el proceso de acumulación de los depósitos arqueológicos del área de estudio. Además de la utilidad y eficacia de las técnicas geofísicas, enfoque principal del presente trabajo, podemos señalar que en PIN7 se logró identificar que el volumen mayor del montículo fue acumulado durante el Formativo Tardío. De esta manera, a partir de las anomalías identificadas con base en las técnicas presentadas a lo largo de este trabajo, se identificaron en el centro del montículo (S2E14) una serie de elementos de cocción sobrepuestos con fragmentos grandes de cerámica del Formativo Tardío. Asimismo, las anomalías identificadas a las orillas del sitio, en el lado oeste, estaban asociadas a actividades pirotecnológicas del Clásico Terminal sobrepuestas a depósitos del Formativo Tardío, junto con fechas de $\mathrm{C}^{14}$ que comprueban ambas épocas de ocupación.

\section{Agradecimientos}

Nos gustaría agradecer a la National Science Foundation por haber otorgado la beca BCS-1115361 a Hector Neff. Asimismo, a los arquéologos y asistentes que han formado parte del PACS, especialmente a Tim Garfin, Sachiko Sakai, Paul Burger y Jorge Hernández. De igual manera, a los investigadores Brendan Culleton, Vera Tiesler y Andrea Cucina por su apoyo, así como también a los revisores cuyos comentarios fueron muy enriquecedores para nosotros. 


\section{Bibliografía}

Breiner, Sheldon y Michael D. Coe

1972 "Magnetic Exploration of the Olmec Civilization: Magnetic Surveys Have Been Highly Successful in Locating Olmec Monuments at the Site of the Oldest Known Civilization in Mesoamerica”, American Scientist, 60 (5): 566575 .

Bruhns, Karen Olsen

1980 "Plumbate Origins Revisited", American Antiquity, 45 (4): 845-848. DOI: https: //doi.org/10.2307/280154.

Canuto, Marcello A., Francisco Estrada-Belli, Thomas G. Garrison, Stephen D. Houston, Mary Jane Acuña, Milan Kováč, Damien Marken, Philippe Nondédéo, Luke Auld-Thomas y Cyril Castanet

2018 "Ancient Lowland Maya Complexity as Revealed by Airborne Laser Scanning of Northern Guatemala”, Science, 361 (Issue 6409, eaau0137). DOI: https: // doi.org/10.1126/science.aau0137.

Chase, Arlen F., Diane Z. Chase y John F. Weishampel

2013 "The Use of LIDAR at the Maya Site of Caracol, Belize", Mapping Archaeological Landscapes from Space, pp. 187-197, Douglas C. Comer y Michael J. Harrower (eds.). Baltimore: Springer. DOI: https://doi.org/10.1007/978-1-4614-6074-9.

Chinchilla Mazariegos, Oswaldo, Víctor Castillo, Carl Lipo, Hector Neff, Kristin N. Safi, Clarus Backes, Veronica Harper, Marisela Galindo y James T. Daniels

2008 "Las calzadas de Cotzumalguapa: nuevos datos arqueológicos y geofísicos", XXI Simposio de Investigaciones Arqueológicas en Guatemala, pp. 1237-1258, J. P. Laporte, B. Arroyo y H. Mejía (eds.). Guatemala: Museo Nacional de Arqueología y Etnología.

Coe, Michael D. y Kent V. Flannery

1967 Early Cultures and Human Ecology in South Coastal Guatemala. Washington, D.C.: Smithsonian Institution Press (Smithsonian Contributions to Anthropology, 3).

Cox, Chris

1992 "Satellite Imagery, Aerial Photography and Wetland Archaeology. An Interim Report on an Application of Remote Sensing to Wetland Archaeology: The Pilot Study in Cumbria, England", World Archaeology, 24 (2): 249-267. DOI: http://doi.org/10.1080/00438243.1992.9980206.

Daniels, James T., Margarita Cossich, Hector Neff y Barbara Arroyo

2012 "Las investigaciones geofísicas en el sitio de Kaminaljuyú", XXV Simposio de Investigaciones Arqueológicas en Guatemala, pp. 537-549, Barbara Arroyo, Lorena Paiz y Héctor Mejía (eds.). Guatemala: Museo Nacional de Arqueología y Etnología, Guatemala. 
Fowler Jr., William R. y Howard H. Earnest Jr.

1985 "Settlement Patterns and Prehistory of the Paraiso Basin of El Salvador", Journal of Field Archaeology, 12 (1): 19-32.

Goodman, Dean

2017 GPR-Slice v. 7.0. Ground Penetrating Radar Imaging Software.

Healy, Paul y Mary Pohl

1980 Archaeology of the Rivas Region, Nicaragua. Waterloo: Wilfrid Laurier University Press.

Jadot, Elsa, Grégory Pereira, Hector Neff y Michael D. Glascock

2019 "All that Glitters is Not Plumbate: Diffusion and Imitation of Plumbate Ceramics during the Early Postclassic Period (AD 900 - 1200) at the Malpaís of Zacapu, Michoacán, México", Latin American Antiquity, 30 (2): 318-332.

Kennett, Douglas J., Dolores R. Piperno, John G. Jones, Hector Neff, Barbara Voorhies, Megan K. Walsh y Brendan J. Culleton

2010 "Pre-Pottery Farmers on the Pacific Coast of Southern Mexico", Journal of Archaeological Science, 37 (12): 3401-3411. DOI: https://doi.org/10.1016/ j.jas.2010.07.035.

Kowalski, Jeff K., Alfredo Barrera Rubio, Heber Ojeda Más y José Huchím Herrera 1993 "Archaeological Excavations of a Round Temple at Uxmal: Summary Discussion and Implications for Northern Maya Culture History", Eighth Palenque Round Table, pp. 281-296, Martha J. Macri, Jan McHargue y Merle Greene Robertson (eds). San Francisco, CA: Pre-Columbian Art Research Institute.

Love, Michael

2002 Early Complex Society in Pacific Guatemala: Settlements and Chronology of the Rio Naranjo, Guatemala. Provo: Brigham Young University (Papers of the New World Archaeological Foundation, 66).

Love, Michael W., Marion Popenoe de Hatch y Héctor L Escobedo

2002 "Ceramic Chronology of Preclassic Period Western Pacific Guatemala and Its Relationship to Other Regions", Incidents of Archaeology in Central America and Yucatán: Essays in Honor of Edwin M. Shook, pp. 51-73, Edwin Shook, Michael Love, Marion Popenoe Hatch y Héctor L. Escobedo (eds.). Lanham: University Press of America.

Lowe, Gareth W., Thomas A. Lee Jr. y Eduardo Martínez Espinosa

1982 Izapa: An Introduction to the Ruins and Monuments. Provo: Brigham Young University, Provo, UT (Papers of the New World Archaeological Foundation, 31).

Medellín Zenil, Alfonso

1960 Cerámicas del Totonacapan: exploraciones arqueológicas en el centro de Veracruz. Xalapa: Universidad Veracruzana, Instituto de Arqueología. 
Mountjoy, Joseph B.

1991 "West Mexican Stelae from Jalisco and Nayarit", Ancient Mesoamerica, 2 (1):

21-33. DOI: https://doi.org/10.1017/S0956536100000341.

Nance, C. Roger

1992 "Guzmán Mound: A Late Preclassic Salt Works on South Coast of Guatemala”, Ancient Mesoamerica, 3 (1): 27-46. DOI: https://doi.org/10.1017/ S0956536100002273.

Neff, Hector

1984 "The Developmental History of the Plumbate Pottery Industry in the Eastern Soconusco Region, AD 600 through AD 1250", tesis doctoral en Antropología. Santa Barbara: University of California.

1989 "The Effect of Interregional Distribution on Plumbate Pottery Production", Ancient Trade and Tribute: Economies of the Soconusco Region of Mesoamerica, pp. 249-267, Barbara Voorhies (ed.). Provo: University of Utah Press.

2002 "Sources of raw material used in plumbate pottery", Incidents of Archaeology in Central America and Yucatan: Essays in Honor of Edwin M. Shook, pp. 217-231, Edwin Shook, Michael Love, Marion Popenoe Hatch y Héctor L. Escobedo (eds.). Lanham: University Press of America.

2003 "Analysis of Mesoamerican Plumbate Pottery Surfaces by Laser Ablation-Inductively Coupled Plasma-Mass Spectrometry (LA-ICP-MS)", Journal of Archaeological Science, 30 (1): 21-35. DOI: https://doi.org/10.1006/jasc.2001.0801.

2012 Informe de la primera temporada de campo del Proyecto Arqueológico Costa del Soconusco. México: Instituto Nacional de Antropología e Historia.

Neff, Hector, Debora M. Pearsall, John G. Jones, Bárbara Arroyo, Shawn K. Collins y Dorothy E. Freidel

2006 "Early Maya Adaptive Patterns: Mid-Late Holocene Paleoenvironmental Evidence from Pacific Guatemala”, Latin American Antiquity, 17 (3): 287-315. DOI: https://doi.org/10.2307/25063054.

Neff, Hector, Paul H. Burger, Brendan J. Culleton, Douglas J. Kennett y John G. Jones 2018 "Izapa's Industrial Hinterland: The Eastern Soconusco Mangrove Zone during Archaic and Formative Times", Ancient Mesoamerica, 29 (2): 395-411. DOI: https://doi.org/10.1017/S0956536118000299.

Pope, Kevin O. y Bruce H. Dahlin

1989 "Ancient Maya Wetland Agriculture: New Insights from Ecological and Remote Sensing Research", Journal of Field Archaeology, 16 (1): 87-106. DOI: https:// doi.org/10.1179/jfa.1989.16.1.87.

Rosenswig, Robert M., Ricardo López-Torrijos, Caroline E. Antonelli y Rebecca R. Mendelsohn 2013 "Lidar Mapping and Surface Survey of the Izapa State on the Tropical Piedmont of Chiapas, Mexico", Journal of Archaeological Science, 40 (3): 14931507. DOI: https://doi.org/10.1016/j.jas.2012.10.034.

Rosenswig, Robert M., Ricardo López-Torrijos y Caroline E. Antonelli

2015 "Lidar Data and the Izapa Polity: New Results and Methodological Issues 
from Tropical Mesoamerica”, Archaeological and Anthropological Sciences, 7 (4): 487-504. DOI: https://doi.org/10.1007/s12520-014-0210-7.

Safi, Kristin N., Oswaldo Chinchilla, Carl P. Lipo y Hector Neff

2012 "Using Ground-Penetrating Radar to Examine Spatial Organization at the Late Classic Maya Site of El Baúl, Cotzumalhuapa, Guatemala”, Geoarchaeology, 27(5): 410-425. DOI: https://doi.org/10.1002/gea.21414.

Scollar, Irwin, Alain Tabbagh, Albert Hesse e Irmela Herzog

1990 Archaeological Prospecting and Remote Sensing. Cambridge: Cambridge University Press.

Shepard, Anna Osler

1948 Plumbate, A Mesoamerican Trade Ware. Washington, D.C.: Carnegie Institution of Washington.

Shook, Edwin M.

1965 "Archaeological Survey of the Pacific Coast of Guatemala", Handbook of Middle American Indians, vol. 2, pp. 180-194, G. R. Willey (ed.). Austin: University of Texas Press.

Snarskis, Michael J. y Silvia Salgado

1986 "The Stratigraphic Excavation of Plumbate Pottery at the Nacascolo Site, Costa Rica”, Mexicon, 8(6): 128-132.

Valdés, Juan Antonio y Jonathan Kaplan

2000 "Ground-Penetrating Radar at the Maya Site of Kaminaljuyu, Guatemala", Journal of Field Archaeology, 27(3): 329-342. DOI: https://doi.org/10.1179/ jfa.2000.27.3.329.

Voorhies, Barbara

1976 The Chantuto People: An Archaic Period Society of the Chiapas Littoral, México. Provo: Brigham Young University (Papers of the New World Archaeological Foundation, 41).

Marx Navarro Castillo. Mexicano. Doctor en Antropología por la Universidad Estatal de Nueva York. Adscrito a la Universidad de Ciencias y Artes de Chiapas en el Departamento de Humanidades, sus investigaciones se centran en la producción cerámica y la especialización artesanal, y desarrolla actualmente el "Proyecto Arqueológico Costa de Soconusco”. Entre sus publicaciones recientes se encuentran "Economic Growth in Mesoamerica: Obsidian Consumption in the Coastal Lowlands", "The Maya Graffiti of Plan de Ayutla, Chiapas" y "Estudios recientes en el Soconusco", todas en coautoría.

marx.navarro@unicach.mx 
Hector Neff. Estadounidense. Doctor en Antropología por la Universidad de California en Santa Bárbara. Adscrito a la Universidad Estatal de California, en Long Beach. Sus especialidades son la arqueometría y la teoría evolutiva, y desarrolla actualmente el "Proyecto Arqueológico Costa de Soconusco". Entre sus publicaciones más recientes se encuentran "Characterization of Archaeological Sediments Using FTIR and PXRF: An Application to Formative Period Pyroindustrial Sites in Pacific Coastal Southern Chiapas, Mexico", "Assessing the provenance of Poverty Point Copper through LA-ICP-MS Compositional Analysis" e "Izapa's Industrial Hinterland: The Eastern Soconusco Mangrove Zone during Archaic and Formative Times", todas en coautoría.

hector.neff@csulb.edu 